incidence of depression in the Thai elderly. J Med Assoc Thai 2002;85:540-544. 6. Unutzer J. Diagnosis and treatment of older adults with depression in primary care. Biol Psychiatry 2002;52:285-292.

7. Brown MN , Lapane KL, Luis A F.The management of depression in older nursing home residents. J Am Geriatr Soc 2002;50: 69-76.

8. Penninx BW, Guralnik JM, Ferrucci L, Simonsick EM, D eeg DJ,W allace RB. D epressive symptoms and physical decline in communitydwelling older persons. JAMA 1998;279: 1720-1726.

9. W agner FA, Gallo JJ, D elva J. D epression in late life:A hidden public health problem for Mexico? Salud Publica Mex 1999;41:189-202. 10. Yesavage JA, Brink TL, Rose TL, Lum 0, Huang V,Adey M et al. D evelopment and validation of a geriatric depression screening scale:A preliminary report.J Psychiatr Res 1982-83;17:37-49.

11. Chan AC. C linical validation of the Geriatric Depression Scale (GDS): C hinese version. J A ging Health 1996;8:238-253.

12. Fernández-San Martín MI,Andrade C, Molina J, Muñoz PE, Carretero B, Rodríguez $M$ et al. Validation of the Spanish version of the geriatric depression scale (GDS) in primary care. Int J Geriatr Psychiatry 2002;17:279-287.

13. Pichot P, López-Ibor A liño JJ,Valdés-Miyar M. DSM-IV. Manual diagnóstico y estadístico de los trastornos mentales. Barcelona: Masson, SA;1995. 14. Snowdon J, D onnelly N .A study of depression in nursing homes. J Psychiatr Res 1986;20:327-333.

15.Teresi J,A brams R, Holmes D, Ramírez M, Eimicke J. Prevalence of depression and depression recognition in nursing homes. Soc Psychiatry Psychiatr Epidemiol 2001;36:613-620. 16. Parmelee PA, Katz IR, Lawton MP. Depression among institutionalized aged: Assessment and prevalence estimation. J Gerontol 1989;44:M22-29.

\section{Prevención de la isoinmunización materna al antígeno RhD}

Señor editor: hemos leído con interés el artículo de los doctores Hernández y Ahued, Sangrado transvaginal durante el embarazo, como factor de riesgo para isoinmunización al antígeno Rhesus- $D^{1}$ y nos permitimos presentar las siguientes consideraciones:

Junto al legado científico y la vasta experiencia aportada a la medicina mexicana por el Dr. Luis Sentíes Gutiérrez ${ }^{2}$ en lo referente a la isoinmunización materna al antígeno $\mathrm{RhD}^{3-6}$ esta publicación tiene el mérito de formar parte de la muy escasa literatura nacional sobre el tema de inmunoprofilaxis en mujeres $\mathrm{Rh}$ negativo en la edad reproductiva publicada en los últimos 20 años.

Las prácticas en otros países ${ }^{7}$ con la detección de la hemorragia feto-materna (HFM), como criterio para el empleo de la gamma-globulina anti-D ( $g$-g anti-D), han sido distintas a las nuestras. En México no se tiene experiencia alguna en la cuantificación de la HFM en cualquier etapa del embarazo, ni con la prueba de Kleihauer-Betke (PKB). Cierto es que se describe como una técnica muy sensible, pero es poco reproducible y muy imprecisa para cuantificar la HFM, ${ }^{7}$ debido a las múltiples fuentes de error que dificultan su interpretación (características del frotis de sangre, número de eritrocitos evaluados, células fetales no teñibles, variaciones en el $\mathrm{pH}$ del reactivo, entre otras). Además de que no permite distinguir la hemoglobina fetal presente en los eritrocitos maternos que llegan a ser cercanos a $6 \%$, o diferenciar la $\mathrm{Hb}$ fetal de los pacientes con talasemia o enfermedad de células falciformes, ${ }^{7}$ condiciones que generan resultados falsos positivo. Aun las técnicas más recientes ${ }^{8}$ tienen un coeficiente de variación entre $15-20 \%$, de acuerdo con el nivel de detección. No ha sido sometido a validez el empleo de las pruebas para la cuantificación de la HFM en embarazos menores a las 20 semanas de gestación, por lo que no se considera una prueba útil en estas condiciones. Tampoco hay evidencia alguna que muestre las ventajas en la prevención de la isoinmunización al emplear la cuantificación de la HFM (independientemente de la técnica) contra el uso de los criterios clínicos comentados. Es decir, que en ausencia de la experiencia o infraestructura para realizar la cuantificación de la HFM, ésta no debe ser aún un criterio para ser incorporada dentro de las medidas de prevención de la isoinmunización.

Es una práctica efectiva y recomendada $^{9-12}$ la utilización posparto o posa- borto de las dosis de g-g anti-D (300 o $150 \mathrm{ug}$ ). Esta cantidad de anti-D es capaz de prevenir la sensibilización de 30-15 ml, de sangre fetal presente en la circulación materna. ${ }^{13}$ Aproximadamente $0.4 \%$ de las gestantes tienen HFM mayor a $4 \mathrm{ml}$. Cerca de $0.3 \%$ reciben más de $30 \mathrm{ml}$. de sangre fetal y no serán protegidas ni con 300 ni con 150 ug de g-g anti-D. Estos casos especiales, generalmente secundarios a traumatismos abdominales o transfusión eritrocitaria $\mathrm{RhD}$, deberán recibir cantidades adicionales de g-g anti-D, calculadas con base en la cuantificación de la HFM mediante la PKB. ${ }^{7,13}$

Los reportes sobre los resultados de la inmunoprofilaxis señalados en el ámbito internacional ${ }^{9,10}$ muestran la reducción del riesgo de isoinmunización en primíparas (RR 0.04 IC 95\% 0.02-0.06) y en el siguiente embarazo (RR 0.12, IC 95\% 0.07-0.23), aplicando el biológico en las primeras 72 horas del posparto y, más recientemente, en dos dosis con diferentes esquemas (semanas 28 y 34 y posparto). Sin embargo, en México se incluyen diferentes modalidades de la conclusion obstétrica, aborto o parto, con el empleo de $150 \mathrm{mg}$ y con resultados similares. ${ }^{5}$ El empleo de la g-g anti-D, en las semanas 28 y 34 , ha demostrado un efecto marginal y no significativo en la reducción del riesgo en mujeres $\mathrm{Rh}$ negativo primíparas o sin distingo de la paridad de $0.30(0.22-0.38)$ y $0.34(0.28$ $0.40)$, respectivamente. ${ }^{14}$

La experiencia internacional ${ }^{11,15} \mathrm{y}$ también en México, ${ }^{5}$ señala que los dos motivos más frecuentes de la sensibilización materna son la no aplicación de la g-g anti-D en sus embarazos previos $^{11,12}$ o la falta de cumplimiento de las guías o lineamientos del programa; mientras que son menos frecuentes las razones socioeconómicas, la baja disponibilidad del biológico en el mercado y las condiciones biológicas (respuesta imunológica individual, momento de la sensibilización, etcétera).

En la cohorte de mujeres Rh negativo del Instituto Nacional de Perinatología (INPer), se identificaron 629 
mujeres sensibilizadas. El 86.2\% de ellas acudieron a su atención médica ya con el problema de isoinmunización y 3.5\% de las que se sensibilizaron a pesar de la aplicación de la g-g anti-D. Estas gestantes tenían problemas obstétricos adicionales, como preeclampsia o embarazo gemelar, mientras que en menos de $20 \%$ de los casos se isoinmunizarán a pesar de las medidas preventivas correctas. ${ }^{5}$ Estos factores de riesgo están presentes en aquellas maternidades donde no hay un procedimiento establecido y regulado para la atención de este tipo de mujeres. ${ }^{11,12,14}$

La disponibilidad de la g-g anti-D, aunque representó un grave problema en la década de los ochenta, ${ }^{3,4}$ se ha modificado en el transcurso de los años, pues la lista de fabricantes participantes en el mercado nacional favoreció sustancialmente la oferta del biológico, quedando limitado el problema a su precio elevado.

Pero, sin duda alguna, el elemento más preocupante es la falta de un marco normativo o regulatorio, pues el INPer es la única institución donde se tienen claramente establecidas las normas y los criterios para la inmunoprofilaxis en la gestante Rh negativo. En el ámbito nacional, la Norma Oficial Mexicana (NOM) para la atención de la mujer durante el embarazo, parto o puerperio y del recién nacido, ${ }^{16}$ señala criterios generales para la atención de la gestante $\mathrm{Rh}$ negativo. No especifica ningún criterio adicional, ni para modificar la dosis, ni la aplicación prenatal o ningún otro procedimiento que permita proteger a las gestantes Rh negativo en riesgo bajo circunstancias clínicas que favorecen la isoinmunización. Por otro lado, la NOM para la disposición de sangre humana y sus componentes ${ }^{17}$ proporciona las recomendaciones sobre los aspectos técnicos y de laboratorio que regulan los criterios para la aplicación profiláctica de la g-g anti-D. Sin embargo, las nuevas propuestas para la actualización de la NOM para la disposición de sangre humana y sus componentes han eliminado cualquier recomendación respecto a las prácticas de inmunoprofilaxis. La tendencia a desaparecer el marco regulatorio de control no es una buena medida epidemiológica.

Los estudios de análisis de costos son abrumadores, la aplicación de la g-g anti-D (aun en el esquema de dos dosis) no supera los 300 dólares americanos. La atención de una mujer Rh negativo isoinmunizada, con enfermedad hemolítica fetal o neonatal grave, rebasa los 30000 dólares americanos. ${ }^{14}$

Bajo una estimación conservadora, de acuerdo con las medidas de prevención en el INPer, se espera que en nuestro país cada año se sensibilicen entre 25 a 50 mujeres en el primer o segundo embarazo, respectivamente, a pesar de haber recibido la profilaxis adecuada bajo las políticas actuales.

\section{Dr Héctor A Baptista González, QCB Fany Rosenfeld Mann. Hematología Perinatal, Instituto Nacional de Perinatología. México, DF, M éxico. baptista@ infosel.net.mx}

\section{Referencias}

1. Hernández AE,A hued AR. Sangrado transvaginal durante el embarazo, como factor de riesgo para isoinmunización al antígeno Rhesus-D. Salud Publica Mex 2003;45:492-496. 2. Cepeda AG, Sentíes L, Fior relli FE, G arcete JA. Prevention of post-partum Rh-factor isoinmunization. Experience of the Hospital $1^{\text {st }}$ Gynecological-O bstetrical of the Mexican Institute of Social Security. Ginecol 0 bstet Mex 1975;37:83-91.

3. Zavala C, Velázquez-Ferrari MA, N avar rete C, Rosales-C orona J, Lisker R. Estimation of the number of females at risk of isoimmunization to the Rho (D) antigen in a sample of the population attended at the Instituto Mexicano del Seguro Social. Arch Invest Med (Mex) 1983;14:199-206.

4. Zavala C, Salamanca F. Mothers at risk of alloimmunization to the $R h(D)$ antigen and availability of gamma-globulin at the Mexican Institute of Social Security. Arch Med Res 1996 Autumn;27(3):373-376.

5. Baptista GHA, Rosenfeld MF, Leiss MT.

Prevention of maternal $R h D$ isoimmunization with anti-D gamma isoinmmunization. Salud Publica Mex 200;43:52-58.

6. Avalos CJ, Díaz EJC, Bravo SJ, Ruiz VV, ZárateSal $H$,Ayllon-Solórzano E et al. Results obtained in the management of materno-fetal isoimmunization to the $\mathrm{Rh}$ factor in the Hospital de Gineco-O bstetricia N 0.3 of the Instituto Mexicano del Seguro Social. Ginecol 0 bstet Mex 1973:34:429-443.

7.W orking party BC SH Blood Transfusion. The estimation of fetomaternal haemorrhage.

Transfusion Med 1999;9:87-92.

8. 0 chsenbeinilmhof $N, O$ chsenbein AF, Seinfert B, Huch A, Huch R, Zimmermann R.

$Q$ uantification of fetomaternal hemorrhage by fluorescence microscopy is equivalent to flow cytometry. Transfusion 2002;42:947-953.

9. Crowther C, Middleton P.Anti-D

administration after childbirth for preventing Rhesus alloimmunization. Cochrane Database Syst Rev 2000;(2):CD 000021.

10. Crowther CA, Keirse MJ.Anti-D administration in pregnancy for preventing rhesus alloimmunization. Cochrane Database Syst Rev 2000;(2): CD 000020.

11. A nonymous. American College of $O$ bstetrician and $\mathrm{G}$ inecologist. practice bulletin. Prevention of Rh $D$ alloinmmunization. N umber 4, May 1999 (replaces educational bulletin N umber 147, 0 ctober 1990). C linical management guidelines for obstetrician Gynecologist. Int I Gynaecol O bstet 1999 Jul;66(1):63-70.

12. Lee D, Contreras M, Robson SC, Rodeck $\mathrm{CH}$, W hittle MJ. Recommendations for the use of anti-D immunoglobulin for Rh prophylaxis. British Blood Transfusion Society and the Royal College of 0 bstetricians and $\mathrm{G}$ ynaecologists. Transfus Med 1999:9:93-97.

13. Bichler J, Schondorfer G, Pabst G, Andresen I. Pharmacokinetics of anti-D IgG in pregnant RhD-negative women. BJO G 2003;110:39-45. 14. C hilcott J, Lloyd JM, W ight J, Forman $\mathrm{K}$, W ray J, Beverly $C$ et al. A review of the clinical effectiveness and cost-effectiveness and costeffectiveness of routine anti-D prophylaxis for pregnant women who are rhesus-negative. Health Technol Assess 2003;7:iii-62.

15. Bowman J.Thirty-five years of Rh prophylaxis. Transfusion 2003;43:1661-1666. 16. Secretaría de Salud. N orma 0 ficial Mexicana N 0 M-007-SSA2-1993,Atención de la mujer durante el embarazo, parto y puerperio y del recién nacido. Criterios y procedimientos para la prestación del servicio. México, DF: D iario O ficial de la Federación, 6 de enero de 1995. Secretaría de Salud. N orma 0 ficial Mexicana N O M-003-SSA2-1993, para la disposición de sangre humana y sus componentes con fines terapéuticos. México, DF: Diario 0 ficial de la Federación, 18 de Julio de 1994. 